\title{
Characterization of Two Types of Silanol Groups on Fused-Silica Surfaces Using Evanescent-Wave Cavity Ring-Down Spectroscopy
}

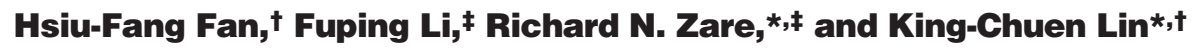 \\ Department of Chemistry, National Taiwan University, and Institute of Atomic and Molecular Sciences, Academia Sinica, \\ Taipei 106, Taiwan, and Department of Chemistry, Stanford University, Stanford, California 94305-5080
}

Evanescent-wave cavity ring-down spectroscopy has been applied to a planar fused-silica surface covered with crystal violet $\left(\mathrm{CV}^{+}\right)$cations to characterize the silanol groups indirectly. A radiation-polarization dependence of the adsorption isotherm of $\mathrm{CV}^{+}$at the $\mathrm{CH}_{3} \mathrm{CN} /$ silica interface is measured and fit to a two-site Langmuir equation to determine the relative populations of two different types of isolated silanol groups. $\mathrm{CV}^{+}$binding at type I sites yields a free energy of adsorption of $-29.9 \pm$ $0.2 \mathrm{~kJ} / \mathrm{mol}$ and a saturation surface density of $(7.4 \pm 0.5)$ $\times 10^{12} \mathrm{~cm}^{-2}$, whereas the values of $-17.9 \pm 0.4 \mathrm{~kJ} / \mathrm{mol}$ and $(3.1 \pm 0.4) \times 10^{13} \mathrm{~cm}^{-2}$ are obtained for the type II sites. The $\mathrm{CV}^{+}$cations, each with a planar area of $\sim 120$ $\AA^{2}$, seem to be aligned randomly while lying over the $\mathrm{SiO}^{-}$ type I sites, thereby suggesting that this type of site may be surrounded by a large empty surface area $\left(>480 \AA^{2}\right)$. In contrast, the $\mathrm{CV}^{+}$cations on a type II sites are restricted with an average angle of $\sim 40^{\circ}$ tilted off the surface normal, suggesting that the $\mathrm{CV}^{+}$cations on these sites are grouped closely together. The average tilt angle increases with increasing concentration of crystal violet so that $\mathrm{CV}^{+}$ cations may be separated from each other to minimize the repulsion of nearby $\mathrm{CV}^{+}$and $\mathrm{SiOH}$ sites.

Adsorption behavior of organic molecules on silica surfaces has been the major theme of interface studies for improving the efficiency of chromatographic separations. When cationic molecules are involved, the strong electrostatic interaction with the negatively charged silanol $(\mathrm{SiOH})$ groups on the surface of the stationary-phase silica may cause unwanted peak broadening and tailing, mainly from a slow kinetic response of the electrostatic adsorption. ${ }^{1-6}$ The surface charge density is one of the primary

* To whom correspondence should be addressed. E-mail: zare@stanford.edu, Fax: 650-723-9262. E-mail: kclin@ccms.ntu.edu.tw. Fax: 886-2-23621483.

${ }^{\dagger}$ National Taiwan University, Taipei 106, and Institute of Atomic and Molecular Sciences, Academia Sinica.

† Stanford University.

(1) Cox, G. B. J. Chromatogr. 1993, 656, 353-367.

(2) Huang, M. X.; Mitchell, D.; Bigelow, M. J. Chromatogr., B 1996, 677, 7784.

(3) Burns, N. L.; Vanalstine, J. M.; Harris, J. M. Langmuir 1995, 11, 27682776.

(4) Huang, M. X.; Plocek, J.; Novotny, M. V. Electrophoresis 1995, 16, 396401.

(5) Huang, M. X.; Dubrovcakova-Schnelderman, E.; Novotny, M. V.; Fatunmbi, H. O.; Wirth, M. J. J. Microcolumn Sep. 1994, 6, 571-576. factors influencing the strength of electrostatics. Accordingly, insight into how the cationic molecules interact with the local silanol groups of the silica surface should aid in the improvement of the design of surface modifications.

Silanol groups play the main role in influencing the interfacial adsorption behavior, possessing an average surface density of $\sim 4.9$ $\times 10^{14} \mathrm{~cm}^{-2}$ on the silica surface ${ }^{7-9}$ or an average surface area of $20.4 \AA^{2}$ per silanol group. As compared to silica sol particles, which have higher surface areas of $(0.1-5) \times 10^{22} \AA^{2} / \mathrm{g},{ }^{7-9}$ only a few studies focus on characterization of silanol groups on a planar silica surface..$^{10-12}$ Ong et al. ${ }^{10}$ first reported that isolated and vicinal silanol groups both exist at the water/silica interface possessing different $\mathrm{p} K_{\mathrm{a}}$ values of 4.9 and 8.5 , with corresponding surface populations of 19 and $81 \%$, respectively. These results were confirmed by means of cross-polarization magic angle spinning $\mathrm{NMR}^{13}$ and fluorescence microscopy. ${ }^{14}$ The isolated silanol groups with $\mathrm{p} K_{\mathrm{a}}=4.9$ are anticipated to be separated far from each other (>5.5 $\AA$ ), permitting proton dissociation. The vicinal silanol groups are located so closely as to form hydrogen bonds directly with their neighbors $(<3.3 \AA)$, which share $46 \%$ of the surface population, or through a water-molecule bridge (3.5-5.5 ̊), which covers $\sim 35 \%$ of the surface population. ${ }^{12,15-17}$

By using second harmonic generation (SHG) with a cationic crystal violet $\left(\mathrm{CV}^{+}\right)$molecular probe to investigate the local density distribution of the isolated silanols $\left(\mathrm{p} K_{\mathrm{a}}=4.9\right)$ on the planar fusedsilica surface, $\mathrm{Xu}$ and co-workers ${ }^{12}$ classified them into two types. The first type of silanol group is anticipated to be surrounded by a large empty surface area $\left(\geq 120 \AA^{2}\right)$ with a surface density of

(6) Ren, F. Y.; Waite, S. W.; Harris, J. M. Anal. Chem. 1995, 67, 3441-3447. (7) Iler, R. K. The Chemistry of Silica; Wiley: New York, 1979; Chapter 6.

(8) Bergna, H. E., Ed. The Colloid Chemistry of Silica; Advances in Chemistry Series 234; American Chemistry Society: Washington, DC, 1994.

(9) Chuang, I. S.; Maciel, G. E. J. Phys. Chem. B 1997, 101, 3052-3064.

(10) Ong S. W.; Zhao, X. L.; Eisenthal, K. B. Chem. Phys. Lett. 1992, 191, 327335.

(11) Huang, X.; Kovaleski, J. M.; Wirth, M. J. Anal. Chem. 1996, 68, 41194123.

(12) Dong, Y.; Pappu, S. V.; Xu, Z. Anal. Chem. 1998, 70, 4730-4735.

(13) Maceil, C. E.; In The Encyclopedia of Nuclear Magnetic Resonance; Grant, D. M., Harris, R. K., Eds.; Wiley: Chichester, UK, 1996; p 4370.

(14) Wirth, M. J.; Ludes, M. D.; Swinton, D. J. Anal. Chem. 1999, 71, 39113917.

(15) Bolis, V.; Cavenago, A.; Fubini, B. Langmuir 1997, 13, 895-902.

(16) Labouriau, A.; Higley, T. J.; Earl, W. L. J. Phys. Chem. 1998, 102, 28972904.

(17) Jeffrey, G. A. An Introduction to Hydrogen Bonding; Oxford University Press: New York, 1997.

10.1021/ac062386n CCC: $\$ 37.00$ @ 2007 American Chemical Society Published on Web 04/13/2007 
$1.1 \times 10^{13} \mathrm{~cm}^{-2}$, whereas the second type includes the rest of the isolated silanol group locating closely with a surface density of $8.2 \times 10^{13} \mathrm{~cm}^{-2}$. Although the SHG technique is a very effective tool in interfacial studies, the interpretation of its results requires that adsorbates on the silica surface must be aligned in a known ordered manner. Aarts et al. ${ }^{18}$ recently used evanescent-wave cavity ring-down spectroscopy (EW-CRDS) as an alternative to characterize the surface structure on atomically smooth hydroxylated amorphous silica and revealed three distinct types of structure by analyzing the near-infrared vibration-combination spectra of surface hydroxyls and adsorbed water species. Two of them essentially correspond to the vicinal silanol groups, being similar but oppositely oriented, and the third one belongs to the isolated silanol group with a quite different orientation. They have thoroughly characterized the $\mathrm{OH}$ modes in the polarized spectra, but lacked knowledge on the detailed distributions of the surface density. Fisk et al. ${ }^{19}$ measured the pH-dependent adsorption isotherms of $\mathrm{CV}^{+}$by a free-running cavity implementation of EWCRDS. The maximum absorbance for each isotherm measured at pH 5.10 and 9.09 can be converted to a ratio of 72.8:27.2 between vicinal and isolated silanol groups, in agreement with the value of 80:20 determined by the SHG technique. ${ }^{20}$ Nevertheless, how the silanol groups are distributed and how the $\mathrm{CV}^{+}$cations are aligned on the silica surface are lacking. In this work, we attempt to characterize the local density distribution and the alignment of dye molecules adsorbed on the surface using similarly an EWCRDS technique. The interfacial adsorbance thus obtained depends on the amount of adsorbates present and their alignment on the surface. The EW-CRDS technique, which combines the CRDS technique with the attenuated total reflectance method, was first developed by Pipino et al. ${ }^{21}$ to probe species at an air/solid interface.

CRDS relies on measurement of the decay rate of light trapped in an optical cavity, confined within two highly reflective mirrors facing each other. ${ }^{22-25}$ When a pulse of laser radiation is guided into the front cavity mirror, the trapped light bounces back and forth between two mirrors, in which only a small fraction of the radiation leaks out of the cavity for each pass. The light decay, which is detected behind the rear mirror, appears exponential with a ring-down time constant that is inversely proportional to the total cavity losses. These losses are caused by the losses of the mirrors and other optics placed within the cavity, light absorption and scattering by the cavity medium, and absorption losses of analytes in optical communication with the cavity. Because the design used for applications of thin films and condensed matter must be modified to minimize cavity losses, the EW-CRDS technique and its analogs emerge about a decade after the development of the

(18) Aarts, I. M. P.; Pipino, A. C. R.; Hoefnagels, J. P. M.; Kessels, W. M. M.; van de Sanden, M. C. M. Phys. Rev. Lett. 2005, 95, 166104-1-166104-4.

(19) Fisk, J. D.; Batten, R.; Jones, G.; O'Reilly, J. P.; Shaw, A. M. J. Phys. Chem. B 2005, 109, 14475-14480.

(20) Tong, Z. G.; Jakubinek, M.; Wright, A.; Gillies, A.; Loock, H. P.; Rev. Sci. Instrum. 2003, 74, 4818-4826.

(21) Pipino, A. C. R.; Hudgens, J. W.; Huie, R. E. Chem. Phys. Lett. 1997, 280, 104-112.

(22) O'Keefe, A.; Deacon, D. A. G. Rev. Sci. Instrum. 1988, 59, 2544-2551.

(23) Lommatzsch, U.; Wahl, E. H.; Owano, T. G.; Kruger, C. H.; Zare, R. N. Chem. Phys. Lett. 2000, 320, 339-344.

(24) Huang, H. Y.; Chuang, W. T.; Sharma, R. C.; Hsu, C. Y.; Lin, K. C.; Hu, C. H. J. Chem. Phys. 2004, 121, 5253-5260.

(25) Scherer, J. J.; Paul, J. B.; O’Keefe, A.; Saykally, R. J. Chem. Rev. 1997, 97, $25-52$. gas-phase CRDS method. ${ }^{18,19,21,26-42}$ The EW-CRDS technique takes advantage of the evanescent wave to monitor interaction between adsorbates and the solid surface. The evanescent wave can be formed at a totally internal reflection interface between an optical medium and a sample, with a penetration depth about half the radiation wavelength. Studies of interfacial phenomena by means of this technique have rapidly attracted wide attention ${ }^{18,19,21,26-42}$ including adsorption behavior at various conditions, ${ }^{26,27}$ and the thermodynamic properties involved, ${ }^{28}$ structure of adsorbed species, ${ }^{18}$ absolute surface coverage, ${ }^{30}$ adsorption constants for monolayer and within the layers, ${ }^{32}$ electrogenerated species monitoring within an electrochemical cell, ${ }^{33}$ hemoglobin adsorption at the interface, ${ }^{34}$ and molecular orientation at the gas/solid and liquid/solid interfaces. ${ }^{30,35}$ It has also been used as an extremely sensitive detector to replace conventional UV-visible detectors in HPLC separations. ${ }^{41,42}$

In this work, the EW-CRDS technique is employed to characterize the isolated silanol groups on a planar fused-silica surface, by using a $\mathrm{CV}^{+}$molecular probe to determine the surface density distributions and its alignment at the $\mathrm{CH}_{3} \mathrm{CN}$ /silica interface. A stream of crystal violet solution is made to flow through a prism base, and $\mathrm{CV}^{+}$species are partially adsorbed on the bare surface. The Langmuir fit of the adsorption isotherms, as acquired in terms of both s- and p-polarized radiation, manifests two different types of isolated silanol distributions. The corresponding surface densities along with the orientation angles of $\mathrm{CV}^{+}$adsorbed on each type surface are evaluated. The general trend of the results is similar to those reported by $\mathrm{Xu}$ and co-workers ${ }^{12}$ using the SHG method. But the details differ from each other and will be thoroughly discussed. In addition, a comparison with the work by Aarts et al. ${ }^{18}$ is also made.

\section{EXPERIMENTAL SECTION}

Figure 1 shows the EW-CRDS apparatus. The optical cavity was confined between two mirrors with high reflectance of $>99.97 \%$ over a broad wavelength range from 583 to $663 \mathrm{~nm}$. The

(26) Shaw, A. M.; Hannon, T. E.; Li, F.; Zare, R. N. J. Phys. Chem. B 2003, 107, 7070-7075.

(27) Fisk, J. D.; Batten, R.; Jones, G.; O’Reilly, J. P.; Shaw, A. M. J. Phys. Chem. B 2005, 109, 14475-14480.

(28) Fan, H. F.; Hung, C. Y.; Lin, K. C. Anal. Chem. 2006, 78, 3583-3590.

(29) Pipino, A. C. R.; Hudgens, J. W.; Huie, R. E. Rev. Sci. Instrum. 1997, 68, 2978-2989.

(30) Pipino, A. C. R.; Hoefnagels, J. P. M.; Watanabe, N. J. Chem. Phys. 2004, 120, 2879-2888.

(31) Pipino, A. C. R. Phys. Rev. Lett. 1999, 83, 3093-3096.

(32) Martin, W. B.; Mirov, S.; Martyshkin, D.; Venugopalan, R.; Shaw, A. M. J. Biomed. Opt. 2005, 10, 024025-1-024025-7.

(33) Mazurenka, M.; Wilkins, L.; Macpherson, J. V.; Unwin, P. R.; Mackenzie, S. R. Anal. Chem. 2006, 78, 6833-6839.

(34) Everest, M. A.; Black, V. M.; Haehlen, A. S.; Haveman, G. A.; Kliewer, C. J.; Neill, H. A. J. Phys. Chem. B 2006, 110, 19461-19468.

(35) Li, F.; Zare, R. N. J. Phys. Chem. B 2005, 109, 3330-3333.

(36) Pipino, A. C. R. Appl. Opt. 2000, 39, 1449-1453.

(37) Hallock, A. J.; Berman, E. S. F.; Zare, R. N. Anal. Chem. 2002, 74, 17411743.

(38) Tarsa, P. B.; Rabinowitz, P.; Lehmann, K. K. Chem. Phys. Lett. 2004, 383, 297-303.

(39) Tarsa, P. B.; Wist, A. D.; Rabinowitz, P.; Lehmann, K. K. Appl. Phys. Lett. 2004, 85, 4523-4525.

(40) Hannon, T. E.; Chah, S.; Zare, R. N. J. Phys. Chem. B 2005, 109, 74357442.

(41) Synder, K. L.; Zare, R. N. Anal. Chem. 2003, 75, 3086-3091.

(42) Bechtel, K. L.; Zare, R. N.; Kachanov, A. A.; Sanders, S. S.; Paldus, B. A. Anal. Chem. 2005, 77, 1177-1182. 


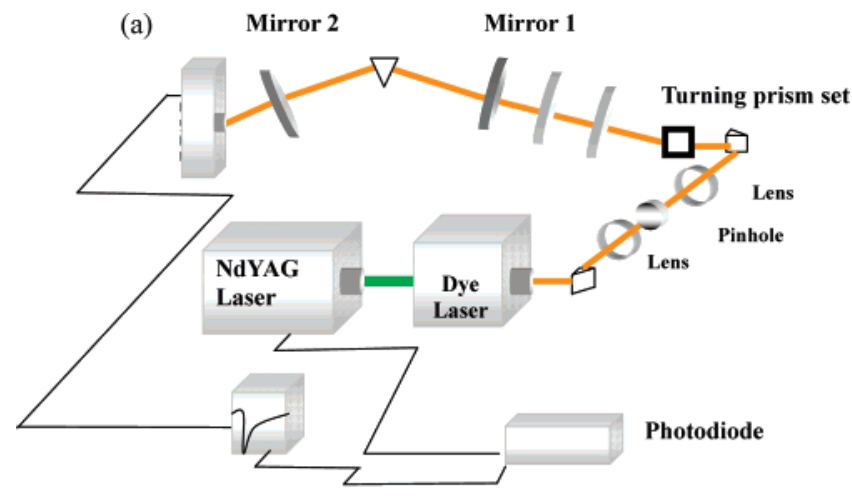

(b)

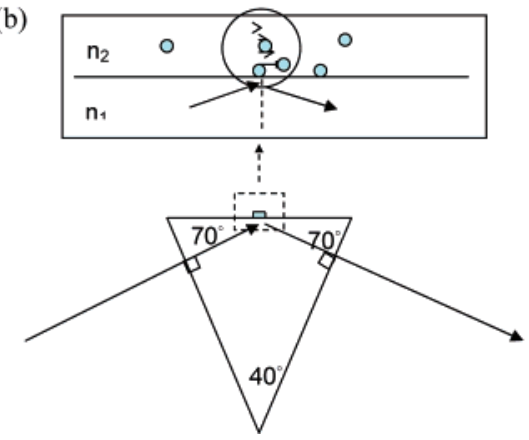

Figure 1. 1. (a) Schematic diagram of the experimental setup for the evanescent-wave cavity ring-down spectroscopy. (b) Enlarged picture of flow cell and prism.

mirrors have a radius of curvature of $100 \mathrm{~cm}$ and a diameter of $2.54 \mathrm{~cm}$ (10CM00SR.30F, Newport). The mirrors were mounted at $\sim 55 \mathrm{~cm}$ apart in such a way that their positions may be slightly adjusted to facilitate optical alignment. A fused-silica prism (Rocky Mountain Instrument), especially designed in the shape of an isosceles triangle with the apex of $40^{\circ}$, was positioned in the center of the cavity. Its side faces were antireflection coated $R<0.012 \%$, while its base was polished to a flatness of $\lambda / 20$ with an rms roughness of $<1 \mathrm{~nm}$. A Teflon flow cell was sealed to the prism base surface with a Viton O-ring, allowing flow of the prepared solutions at $2.2 \mathrm{~mL} / \mathrm{min}$, which was regulated by a piston pump. The flow cell accommodated a volume of $\sim 100 \mu \mathrm{L}$.

The radiation source was a tunable dye laser (PDL-1, SpectraPhysics), which was pumped by the 20-Hz, 5-8-ns pulsed second harmonic of an Nd:YAG laser (DCR, Spectra-Physics), emitting at $600 \mathrm{~nm}$ while operating on rhodamine 610 dye. In order to remain mostly the lowest-order transverse mode $\mathrm{TEM}_{00}$, the pulsed beam was collimated through a spatial filter made of a pair of lenses of 6 - and 15 -cm focal lengths and a 50- $\mu$ m-diameter pinhole. The output light passed through a $\lambda / 2$ wave plate that allows the beam to be either p- or s-polarized, depending on the polarization direction parallel or perpendicular to the incident plane. The purity of polarization is better than $1 \%$. The beam, with the energy controlled to be less than $2 \mathrm{~mJ} /$ pulse, was coupled into the optical cavity. The normals of both mirrors were tilted equally off the axis of the linear cavity, guiding the incident and reflected beams along the mirror normal to propagate perpendicularly through the side surfaces of the prism. The beam entering the prism was totally reflected with evanescent field penetration through the base surface. The penetration depth was estimated to be $366 \mathrm{~nm}$, given the following conditions: the refractive index of 1.457 and 1.3441 for the fused silica and the acetonitrile, respectively, the incidence angle of $70^{\circ}$ at the interface with respect to the surface normal, and the employed laser radiation at $600 \mathrm{~nm}$. The evanescent wave may be absorbed by the $\mathrm{CV}^{+}$adsorbate at the liquid/silica interface. A photomultiplier tube (PMT) (R4632, Hamamatsu) was positioned behind the rear mirror to detect the light intensity that leaks out on each round trip. The signal intensity was inspected to avoid any optical saturation of the PMT. The temporal profile of the ring-down signal was recorded on a transit digitizer (LT342, LeCroy) and transferred to a personal computer. The ring-down time constant may be determined by a best fit of the acquired exponential decay with the aid of a Labview program.

Careful preparation of the prism surface was required to achieve reproducible results. The base surface was first washed with concentrated hydrochloric acid $(5 \mathrm{M})$, followed by washing with deionized water and then with methanol, each flow lasting for $1 \mathrm{~min}$. Crystal violet solutions were prepared in acetonitrile $\left(\mathrm{CH}_{3} \mathrm{CN}\right)$. The dye was purchased (Sigma-Aldrich, St. Louis, MO) and used without any further purification. Its adsorption behavior at the $\mathrm{CH}_{3} \mathrm{CN} /$ silica interface was characterized as a function of concentration and polarization. After the flowing solution had reached equilibrium with the silica surface, up to 100 traces each of the absorption profiles of solvent and solution were acquired. The p-and s-polarized spectra were measured sequentially for both the blank and the sample solutions. The polarization scrambling should be negligible, once the $\mathrm{CV}^{+}$cation was steadily adsorbed at the $\mathrm{CH}_{3} \mathrm{CN} /$ silica interface. A rapid decay of the corresponding ring-down time constant averaged over the 100 traces was found when dye molecules bind to the silanol groups. After each experiment, the prism surface must be cleaned with concentrated hydrochloric acid, followed by deionized water and then methanol, to restore the initial conditions for the next concentrationdependent measurement.

As compared to gas-phase CRDS, the smaller ring-down time constant in EW-CRDS is caused by the light losses within the cavity, which are mainly attributed to mirror losses, prism attenuation and scattering, and polarization-dependent losses. ${ }^{21}$ Given the ring-down time of $\sim 10 \mu$ s at $600 \mathrm{~nm}$ for the empty cavity, the mirror loss per round trip may be estimated to be 0.00037 by the formula

$$
\tau^{\prime}(v)=t_{\mathrm{r}} / L_{\mathrm{m}}
$$

where $\tau^{\prime}$ is the ring-down time, $t_{\mathrm{r}}$ the round-trip time required to travel the cavity distance, and $L_{\mathrm{m}}$ the mirror loss per round trip. After the prism is inserted into the optical cavity, the ring-down time drops dramatically to $900 \mathrm{~ns}$, caused by additional light losses from the prism including bulk absorption and surface scattering. The resultant cavity loss becomes 0.0041 , which has included bulk absorption, surface scattering, and polarization-dependent losses. Among them, the surface scattering loss is estimated to be 0.0007 , while the polarization-dependent loss is $\sim 0.0001$, assuming the prism angle deviates within \pm 5 arc min. ${ }^{21}$ The CRDS measurements are insensitive to intensity variations in the laser pulses, despite pulse-to-pulse fluctuations of $\pm 5 \%$. Taking into account the round-trip time of $3.7 \mathrm{~ns}$, the ring-down time of $900 \mathrm{~ns}$ for a blank solution, and a relative standard deviation of 0.01 in the ring-down 
(a)

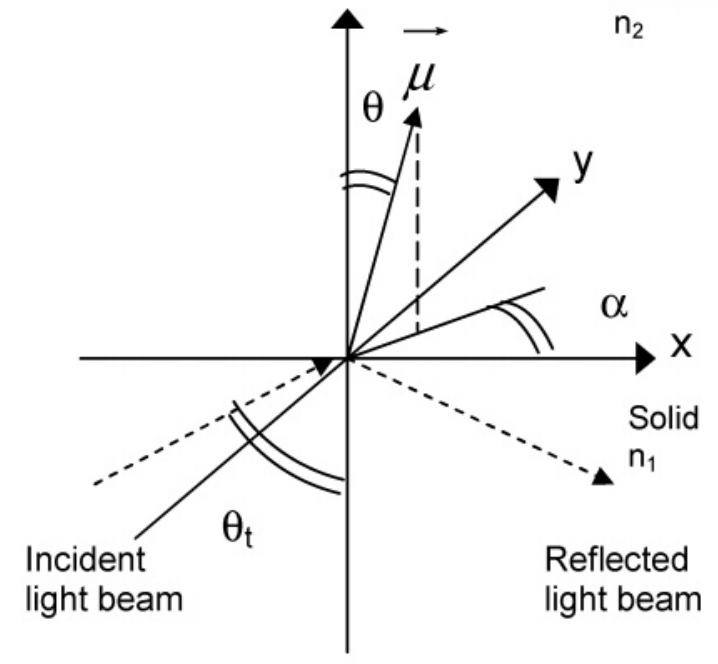

(b)

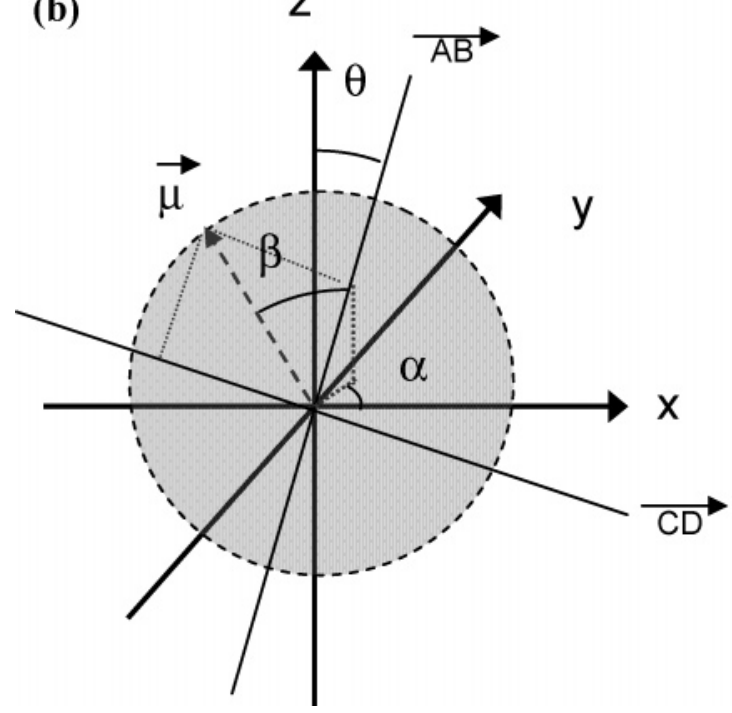

Figure 2. 2. Definition of the coordination system used in the Theory section. The interface is parallel to the $x y$ plane and the incident radiation travels in the $x z$ plane. (a) A transition dipole moment $\mu$ with azimuthal and polar angles $\theta$ and $\alpha$ interacts with the electric field vector of the evanescent wave. (b) The molecular frame of planar crystal violet is defined by the $A B$ and $C D$ plane, in which the $C D$ coordinate is coplanar on the $x y$ plane and the $A B$ coordinate is tilted at an angle $\theta$ from the $z$ axis. The transition dipole moment $\mu$ is confined with the azimuthal angles $\theta, \alpha$, and $\beta$.

time measurements, the minimum detectable absorbance for the current system may be estimated ${ }^{35,42,43}$ to be $5.8 \times 10^{-5}$.

\section{THEORY}

Interfacial absorbance is known to be proportional to the square of the interaction of the transition dipole moment of an adsorbate and the electric field of the evanescent wave. When the excitation radiation is polarized, the corresponding interfacial absorbance can give rise to information on the orientation of the adsorbate on the substrate surface. The alignment of linear methylene blue is used as an example given in Figure $2 \mathrm{a}^{35}$ The interface is defined to be parallel to the $x y$ plane, the incident light

(43) Romanini, D.; Lehmann, K. J. Chem. Phys. 1993, 99, 6287-6301. travels in the $x z$ plane, and the transition dipole moment $\mu$ along the molecular main axis is aligned with azimuthal and polar angles, $\theta$ and $\alpha$. When a rotational symmetry in the distribution of the orientation angle with respect to the surface normal is assumed, the $x$ and $y$ axes become equivalent. Then, the linear transition dipole moment of the molecule may be considered to be symmetric about the surface normal. The orientation distribution function $f(\theta, \alpha)$ is simplified to be $f(\theta) / 2 \pi$ and finally averaged over the azimuthal angle as a measure of the mean orientation angle of the molecule. ${ }^{35}$ Alternatively, the distribution function may be expanded in Legendre polynomials, in which the expansion coefficients are related to the spatial anisotropy parameters. ${ }^{30}$

For our case with the planar molecule, $\mathrm{CV}^{+}$, having the structure symmetry of $D_{3 h}$, the orientation distribution function may be obtained similarly, but in a more complicated manner. As shown in Figure $2 b$, the interface is also defined to be parallel to the $x y$ plane and the light propagation direction is in the $x z$ plane. The $A B$ and $C D$ coordinates define the molecular frame of the planar molecule, with the $\mathrm{CD}$ coordinate coplanar on the $x y$ plane and the $\mathrm{AB}$ coordinate tilted at an angle $\theta$ from the $z$ axis. The transition dipole moments lie perpendicular to one another and only one $\mu$ with the azimuthal angles $\theta, \alpha$, and $\beta$ is displayed in the figure. The component of the transition dipole moment along the $z$ axis is obtained by projecting $\mu$ on the $\mathrm{AB}$ coordinate, followed by projection on the $z$ axis. Its components along the $x$ (or $y$ ) axis may be simultaneously obtained by summation of the $\mu$ projection on the $\mathrm{AB}$ coordinate and then on the $x$ (or $y$ ) axis as well as the $\mu$ projection on the CD coordinate and then on the $x$ (or $y$ ) axis.

When the incident beam is in the $\mathrm{p}$ - (or s-) polarization direction, the interfacial absorbance $A_{\mathrm{p}}\left(\right.$ or $A_{\mathrm{s}}$ ) is associated with the component of the transition dipole moment projected on the $x z$ plane (or on the $y$ axis). The explicit equations for the interfacial absorbance $A_{\mathrm{p}}$ and $A_{\mathrm{s}}$ in terms of different polarization are expressed as

$$
\begin{array}{r}
A_{\mathrm{p}}=a N \int_{0}^{\pi / 2} \sin \theta \mathrm{d} \theta \int_{0}^{2 \pi} \int_{0}^{2 \pi} \mathrm{d} \alpha \mathrm{d} \beta f \\
(\theta, \beta)\left(\left(\mu E_{z} \cos \beta \cos \theta\right)+\left(\mu E_{x}(\cos \alpha \cos \beta \sin \theta+\right.\right. \\
\sin \alpha \sin \beta)))^{2} \\
A_{\mathrm{s}}=a N \int_{0}^{\pi / 2} \sin \theta \mathrm{d} \theta \int_{0}^{2 \pi} \int_{0}^{2 \pi} \mathrm{d} \alpha \mathrm{d} \beta f(\theta, \beta) \\
\left(\left(\mu E_{y}(\sin \alpha \cos \beta \sin \theta+\cos \alpha \sin \beta)\right)\right)^{2}
\end{array}
$$

where $\theta$ is the orientation angle, $f(\theta, \beta)$ is the orientation distribution function, $\alpha$ and $\beta$ are the azimuthal angles, $\mu$ is the transition dipole moment, $E_{i}(i=x, y, z)$ is the electric field component along different axis, $N$ is the surface density of adsorbate at the interface, and $a$ is related with collection efficiency and detection sensitivity. Herein, we assume that the orientation of the adsorbate is isotropic in $\beta$ and $f(\theta, \beta)$ can be replaced by $f(\theta) / 2 \pi$. Then, the above equations become,

$$
\begin{aligned}
& A_{\mathrm{p}}= \frac{a N}{2 \pi} \int_{0}^{\pi / 2} \sin \theta \mathrm{d} \theta \int_{0}^{2 \pi} \int_{0}^{2 \pi} \mathrm{d} \alpha \mathrm{d} \beta f(\theta) \\
&\left(\left(\mu E_{z} \cos \beta \cos \theta\right)+\left(\mu E_{x}(\cos \alpha \cos \beta \sin \theta+\right.\right. \\
&\sin \alpha \sin \beta)))^{2}
\end{aligned}
$$




$$
\begin{aligned}
A_{\mathrm{s}}=\frac{a N}{2 \pi} \int_{0}^{\pi / 2} \sin \theta \mathrm{d} \theta \int_{0}^{2 \pi} \int_{0}^{2 \pi} \mathrm{d} \alpha \mathrm{d} \beta f(\theta) \\
\quad\left(\left(\mu E_{y}(\sin \alpha \cos \beta \sin \theta+\cos \alpha \sin \beta)\right)\right)^{2}
\end{aligned}
$$

Equations 4 and 5 are further simplified by substituting the formula $\left\langle\cos ^{2} \theta\right\rangle=\int_{0}^{\pi / 2} \cos ^{2} \theta f(\theta) \sin \theta \mathrm{d} \theta,^{35}$

$$
\begin{gathered}
A_{\mathrm{p}}=\frac{a \pi N \mu^{2}}{2}\left[2\left\langle\cos ^{2} \theta\right\rangle E_{z}^{2}+\left(2-\left\langle\cos ^{2} \theta\right\rangle\right) E_{x}^{2}\right] \\
A_{s}=\frac{a \pi N \mu^{2}}{2}\left[\left(2-\left\langle\cos ^{2} \theta\right\rangle\right) E_{y}^{2}\right]
\end{gathered}
$$

Finally, an explicit expression for the term of average orientation angle is given by

$$
\left\langle\cos ^{2} \theta\right\rangle=\frac{2 A_{\mathrm{p}} E_{y}^{2}-2 A_{\mathrm{s}} E_{x}^{2}}{2 A_{\mathrm{s}} E_{z}^{2}-A_{\mathrm{s}} E_{x}^{2}+A_{\mathrm{p}} E_{y}^{2}}
$$

The average orientation angle of the planar molecules with respect to the surface normal may be determined accordingly, given the measured interfacial absorbance in terms of polarized radiation, light incidence angle, and the related refractive index of the medium. Note that, for the bound adsorbate molecules, the azimuthal angles, $\alpha$ and $\beta$, are assumed to be isotropic ranging from 0 to $2 \pi$ in the molecular frame, behaving like a free adsorbate. As reported ${ }^{44}$ when $\mathrm{CV}^{+}$is bound to a clay surface, the peak positions of interfacial absorbance around $600 \mathrm{~nm}$ shift only $\sim 1-5 \%$, while the polarization directions of the transition dipole moment remain the same between the free and bound adsorbates. Thus, the $\mathrm{CV}^{+}$cation bound on the silica surface does not seem to show a significant difference from its free cation in the absorption transition. Because the positive charge on the adsorbate delocalizes around the whole planar molecule, the interaction with the negatively charged surface may not favor some fixed point or angle.

In the EW-CRDS experiments, the interfacial absorbance may be expressed as $A=0.434 \kappa l$, according to Beer's law, in which $l$ denotes the penetration depth of the evanescent wave and $\kappa$ is the absorption coefficient. There are two different definitions usually adopted to characterize the penetration depth. One penetration thickness is a measure of the distance at which the electric field falls to 1 /e of its initial value, and the other thickness produces the same absorption if a standard absorption experiment is used..$^{45}$ Either one may not cause different results for the interfacial absorbance as measured in terms of the ring-down time, which is expressed as follows: $: 28,42$

$$
A=\frac{L}{2.303 c}\left(\frac{1}{\tau}-\frac{1}{\tau_{\mathrm{o}}}\right)
$$

Here, $\tau$ and $\tau_{0}$ are the ring-down time as measured with and without adsorbate involved, respectively, $c$ is the light speed, and

(44) Yamaoka, K.; Sasai, R. J. Colloid Interface Sci. 2000, 255, 82-93.

(45) Harrick, N. J. Internal Reflection Spectroscopy; Wiley \& Sons: New York, 1967.

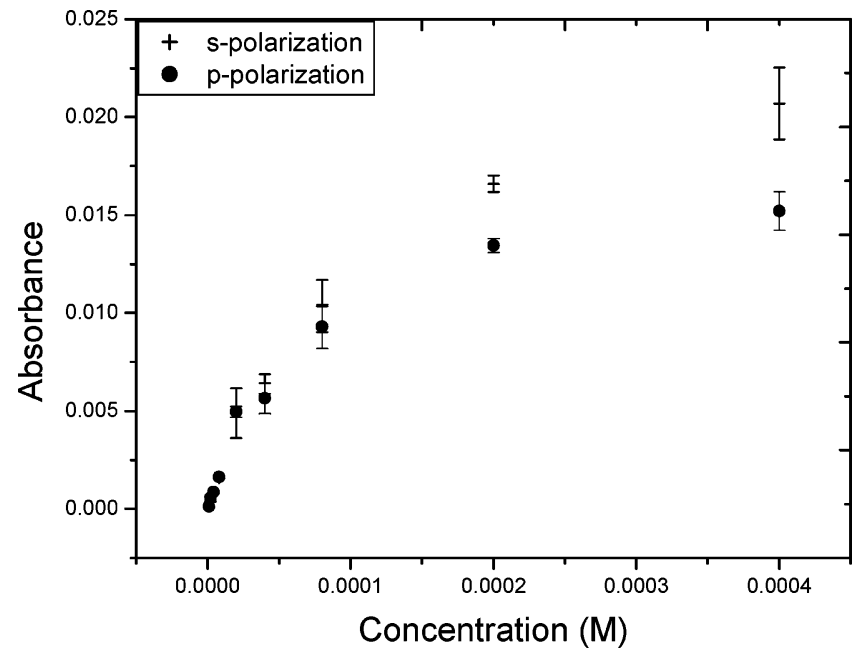

Figure 3. 3. Absorbance of $\mathrm{CV}^{+}$at the $\mathrm{CH}_{3} \mathrm{CN} /$ silica interface versus concentration for $\mathrm{s}$ - and $\mathrm{p}$-polarized radiation systems.

$L$ denotes the length of optical cavity. Note that no knowledge of the penetration depth of the evanescent wave is needed to evaluate eq 9.

\section{RESULTS AND DISCUSSION}

Physical Properties of Adsorption. Figure 3 presents the measurements of the polarization dependence of the adsorption isotherms of $\mathrm{CV}^{+}$at the $\mathrm{CH}_{3} \mathrm{CN}$ /silica interface. The absorbance is evaluated using eq 9 , from the measured ring-down times of the solvent and the sample solution. The radiation polarization makes a significant difference in the interfacial absorbance when the $\mathrm{CV}^{+}$concentration exceeds $(3 \pm 1) \times 10^{-5} \mathrm{M}$. The absorbance with s-polarization becomes larger and the difference grows with increasing concentration. Given the results illustrated in Figure 3 , the average orientation angles of the $\mathrm{CV}^{+}$molecular plane with respect to the surface normal may be evaluated by using eq 8 as described in the next section.

The bulk concentration dependence of the surface density of $\mathrm{CV}^{+}$adsorbed at the $\mathrm{CH}_{3} \mathrm{CN}$ /silica interface may be obtained by the following method. By coating $6 \mu \mathrm{L}$ of $\mathrm{CV}^{+}$at different concentrations between $10^{-7}$ and $10^{-4} \mathrm{M}$ in the methanol solution on the fused-silica surface, a film may be formed at the air/silica interface with a surface area of $\sim 6 \mathrm{~cm}^{2}$, possessing a monolayer surface density from $1.0 \times 10^{11}$ to $1.0 \times 10^{14}$ molecules $/ \mathrm{cm}^{2}$. Given the $A_{\mathrm{p}}$ and $A_{\mathrm{s}}$ measurements as a function of the bulk concentration and the corresponding average orientation angle analyzed by using eq 8 along with evaluation of surface density, the term $a \pi \mu^{2} / 2$ in eqs 6 and 7 may be evaluated, yielding $(2.25 \pm 0.4) \times$ $10^{-16}$ and $(2.24 \pm 0.4) \times 10^{-16}$, respectively, an almost identical result. This term, which is related to the transition probability, is essentially determined from a peak absorption cross section at $600 \mathrm{~nm}$. We assume this term to be the same between air/silica and $\mathrm{CH}_{3} \mathrm{CN}$ /silica interfaces, on which the $\mathrm{CV}^{+}$film is formed, although the conserved quantity is valid only for the integrated absorption cross section. By applying this conservation principle between gas and adsorbed phases, Pipino and co-workers have determined absolute surface coverage with submonolayer sensitivity. ${ }^{30}$

When the film condition is changed to the $\mathrm{CH}_{3} \mathrm{CN} /$ silica interface, the surface density as a function of the bulk concentra- 


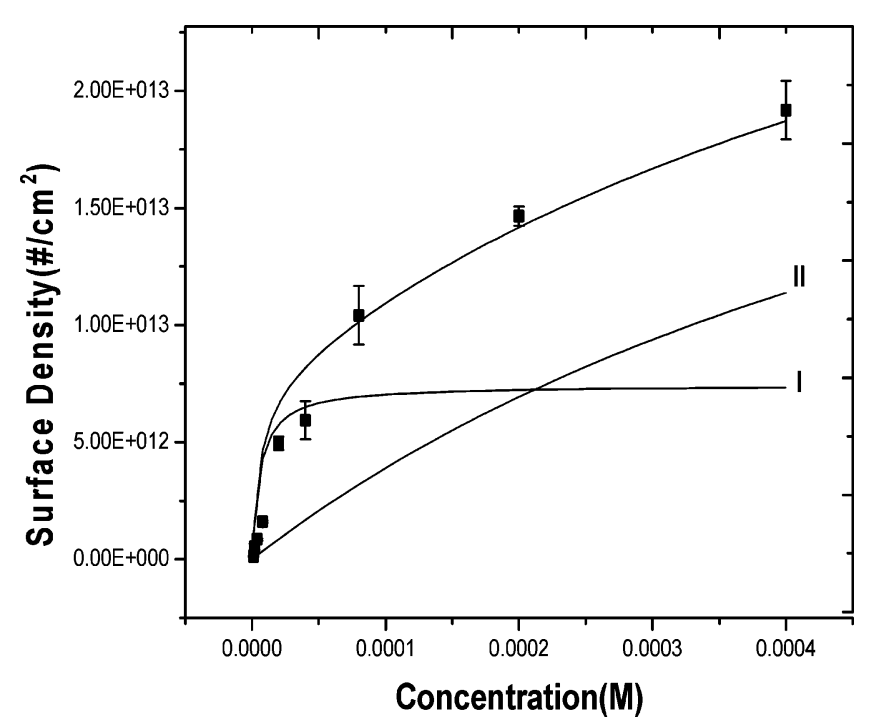

Figure 4. 4. Adsorption isotherm of $\mathrm{CV}^{+}$at the $\mathrm{CH}_{3} \mathrm{CN} /$ silica interface. The adsorption isotherm is fitted by a two-site Langmuir equation, yielding two types of saturation surface densities and adsorption equilibrium constants of $N_{1}^{0}=(7.4 \pm 0.5) \times 10^{12} \mathrm{~cm}^{-2}$ and $K_{\mathrm{l}}=(1.7 \pm 0.2) \times 10^{5} \mathrm{M}^{-1}$ as well as $N_{\|}^{0}=(3.1 \pm 0.4) \times 10^{13}$ $\mathrm{cm}^{-2}$ and $K_{\|}=(1.4 \pm 0.3) \times 10^{3} \mathrm{M}^{-1}$.

\section{Table 1. Saturation Surface Density $\boldsymbol{N}^{\circ}$, Adsorption Equilibrium Constant $K$, and Free Energy of Adsorption $\Delta G$ of $\mathbf{C V}^{+}$Binding on the Type I and Type II Silanol Groups}

\section{type I}

$\begin{array}{ll}N^{\circ} & (7.4 \pm 0.5) \times 10^{12} \mathrm{~cm}^{-2} \\ K & (1.7 \pm 0.2) \times 10^{5} \mathrm{M}^{-1} \\ \Delta \boldsymbol{G} & -29.9 \pm 0.2 \mathrm{~kJ} / \mathrm{mol}\end{array}$

tion may be determined, as shown in Figure 4. Given the $a \pi \mu^{2} / 2$ value determined from the air/silica film experiment, the measurement of $A_{\mathrm{p}}$ and $A_{\mathrm{s}}$ given in Figure 3 , and the corresponding orientation angle determined by eq 8 , the surface density results are best fit by a two-site Langmuir adsorption equation. ${ }^{12}$ That is,

$$
N=N_{\mathrm{I}}^{0} \frac{K_{\mathrm{I}} C}{1+K_{\mathrm{II}} C}+N_{\mathrm{II}}^{0} \frac{K_{\mathrm{II}} C}{1+K_{\mathrm{II}} C}
$$

where $N_{\mathrm{I}}^{0}$ and $N_{\mathrm{II}}^{0}$ denote the saturation surface densities of $\mathrm{CV}^{+}$ binding on the type I and type II silanol groups, respectively; $K_{\mathrm{I}}$ and $K_{\mathrm{II}}$ are the corresponding adsorption equilibrium constants, and $C$ is the bulk concentration of $\mathrm{CV}^{+}$. The fitted values yield $N_{\mathrm{I}}^{0}=(7.4 \pm 0.5) \times 10^{12} \mathrm{~cm}^{-2}$ and $K_{\mathrm{I}}=(1.7 \pm 0.2) \times 10^{5} \mathrm{M}^{-1}$ as well as $N_{\mathrm{II}}^{0}=(3.1 \pm 0.4) \times 10^{13} \mathrm{~cm}^{-2}$ and $K_{\mathrm{II}}=(1.4 \pm 0.3) \times 10^{3}$ $\mathrm{M}^{-1}$. The corresponding free energy of adsorption of $\mathrm{CV}^{+}$may be further determined to be $\Delta G_{\mathrm{I}}=-29.9 \pm 0.2 \mathrm{~kJ} / \mathrm{mol}$ and $\Delta G_{\mathrm{II}}$ $=-17.9 \pm 0.4 \mathrm{~kJ} / \mathrm{mol}$, respectively. For clarity, the results are summarized in Table 1. As shown in Figure 4, the fitting quality based on the two-site Langmuir adsorption equation is reflected by a squared regression coefficient $R^{2}$ of 0.98 . The first and the second terms of eq 10 are also displayed individually, denoted as curves I and II. Apparently, two types of silanol groups exist on the fused-silica surface. For the type I silanol, $\mathrm{CV}^{+}$may bind strongly with the $\mathrm{SiO}^{-}$site via electrostatic attraction, whereas for the type II silanol, $\mathrm{CV}^{+}$also binds with the $\mathrm{SiO}^{-}$site, but is repelled simultaneously by the dipole field of nearby $\mathrm{SiOH}$ groups. Different from this work, when trans-4-[4-(dibutylamino) styryl]1-(3-sulfopropyl)pyridinium and trans-4-[4-(dibutylamino) styryl]1-methylpyridinium iodide, each with a surface area of $\sim 50 \AA^{2}$, were adopted as the molecular probe, only one adsorption equilibrium constant was determined for these two types of silanol sites, because the repulsive force was unimportant for the smaller size molecules. ${ }^{28}$

$\mathrm{Xu}$ and co-workers ${ }^{12}$ had earlier characterized the local density distributions of these two types $\mathrm{SiO}^{-}$sites, which are isolated without any chance to form a hydrogen bond with their neighbors. They verified that the $\mathrm{CV}^{+}$adsorption on both types is via chargecharge attraction by adding triethylamine as a proton acceptor in the solution, thereby obtaining the surface densities for these two types, as mentioned in the introduction. They also obtained the free energies of adsorption of -29.5 and $-12.3 \mathrm{~kJ} / \mathrm{mol}$ for the type I and II sites, respectively.

Both $\mathrm{Xu}$ and co-workers ${ }^{12}$ and we concluded that binding to type I is stronger than to type II, but the values of $\Delta G$ differ for the type II binding in detail. The $\mathrm{CV}^{+}$adsorption on the type I sites shows a stronger attraction and, therefore, larger equilibrium constant and free energy of adsorption, because these silanol sites are widely separated such that the approach of $\mathrm{CV}^{+}$suffers the least hindrance. In contrast, the type II silanol sites are much more closely spaced, and thus, $\mathrm{CV}^{+}$is repelled to a further distance from the $\mathrm{SiO}^{-}$site for reducing the repulsive hindrance caused by the adjacent $\mathrm{SiOH}$ groups. The larger distance between $\mathrm{CV}^{+}$ and the charged surface leads to a smaller electrostatic attraction and, thus, a smaller equilibrium constant and free energy of adsorption.

As for quantitative comparison with the reported results, agreement of $\Delta G$ for the type I sites is excellent, whereas there is $\sim 5 \mathrm{~kJ} / \mathrm{mol}$ difference for the type II sites. The discrepancy could be caused by different analysis methods to extract the $\Delta G$ information. In resolving the adsorption isotherm for the type II sites, we obtained a curve rising very slowly to a plateau (Figure $4)$, from which $K$ and then $\Delta G$ were readily fit, whereas $\mathrm{Xu}$ and co-workers obtained a straight line and then evaluated $\Delta G$ indirectly. Perhaps, the discrepancy can be caused by an additional absorbance contribution from the bulk solution or by electrical double layer effects. As reported, the concentration of adsorbates on the surface is larger by several orders of magnitude than that in the solution, when the bulk solution is under condition of low concentration. ${ }^{28,46}$ Therefore, subtraction of the bulk contribution should lead to a slight and yet negligible change of $\Delta G$. On the other hand, for the cationic adsorbates, the counterions may be adsorbed by the interfacial cations, helping reduce the chargecharge repulsion between the dye cations. The electric potential of the negatively charged surface thus attracts the bulk $\mathrm{CV}^{+}$ cations moving within the evanescent field to enhance the absorbance measurement. Such an effect of electrical double layer may become significant with extra electrolytes added to increase ionic strength of the solution. ${ }^{26,28}$ Once it occurs, the Langmuir equation often fails to fit the adsorption isotherm.

The saturation surface density $N_{\text {II }}^{0}$ determined in this work appears to be consistent with the result of $3.0 \times 10^{13} \mathrm{~cm}^{-2}$ by Xu

(46) Xu, Z.; Li, J.; Dong, Y. Langmuir 1998, 14, 1183-1188. 


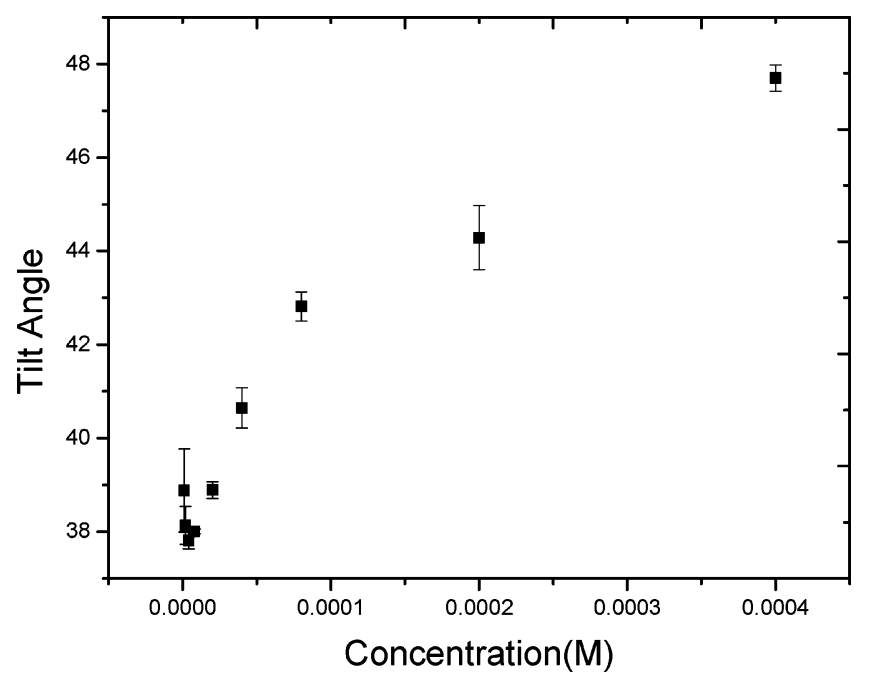

Figure 5. 5. Bulk concentration dependence of orientation angle of $\mathrm{CV}^{+}$at the $\mathrm{CH}_{3} \mathrm{CN} /$ silica interface with respect to the surface normal.

and co-workers ${ }^{12}$ before triethylamine was added as a proton acceptor. Nevertheless, our $N_{\mathrm{I}}^{0}$ value is $\sim 7-8$ times larger than their result of $9.5 \times 10^{11} \mathrm{~cm}^{-2}$. We do not know the source of this discrepancy, but we offer some speculations. The SHG method employed by $\mathrm{Xu}$ and co-workers makes use of the symmetry change of molecules at the interface, being sensitive to the interfacial interaction of molecules and substrate. Nevertheless, the effective application of this technique requires molecules adsorbed on the surface should be aligned in a known ordered manner. If the molecular alignment is random, then information might be lost, as caused by such factors as increased concentration, nature of interfacial interaction, or surface defects. Thus, the SHG method may underestimate the interfacial density of $\mathrm{CV}^{+}$. In contrast, the EW-CRDS method determines the absorbance of the adsorbate depending on its total interfacial density, but free from the alignment restriction because we measure both the sand p-polarized responses.

Orientation of $\mathrm{CV}^{+}$at the $\mathrm{CH}_{3} \mathrm{CN} /$ Silica Interface. Given the interfacial absorbance results from Figure 3, the refractive index of $\mathrm{CH}_{3} \mathrm{CN}\left(n_{2}=1.342\right)$ and fused silica $\left(n_{1}=1.46\right)$, and incident angle of $70^{\circ}$, according to eq 8 , the average orientation angle of $\mathrm{CV}^{+}$with respect to the surface normal is obtained as a function of the concentration. The results are shown in Figure 5. When the concentration is lowered to $10 \mu \mathrm{M}$, the average tilt angle is measured of $\sim 37-38^{\circ}$ and then appears to increase with increased concentration. To confirm the accuracy of measurement, several replicates at lower concentrations were carried out. The beginning tilt angle at low concentration should correspond to the $\mathrm{CV}^{+}$interaction with the type I site alone. Since the binding energy of $\mathrm{CV}^{+}$with the type II site is much smaller, its binding capability is hard to compete at a lower concentration. The average tilt angle at the low concentration is obtained to be $37-38^{\circ}$ relative to the surface normal (Figure 5), which is equivalent to a tilt angle of $52-53^{\circ}$ with respect to the silica surface. Such a result may be fit to the magic angle of $54.7^{\circ}$, while including the uncertainty carried by the assumption of a proportional relationship made between the integrated and the peak absorption cross sections. At this magic angle, the spatial anisotropy parameter becomes zero, and thus, the molecular alignment with respect to the silica
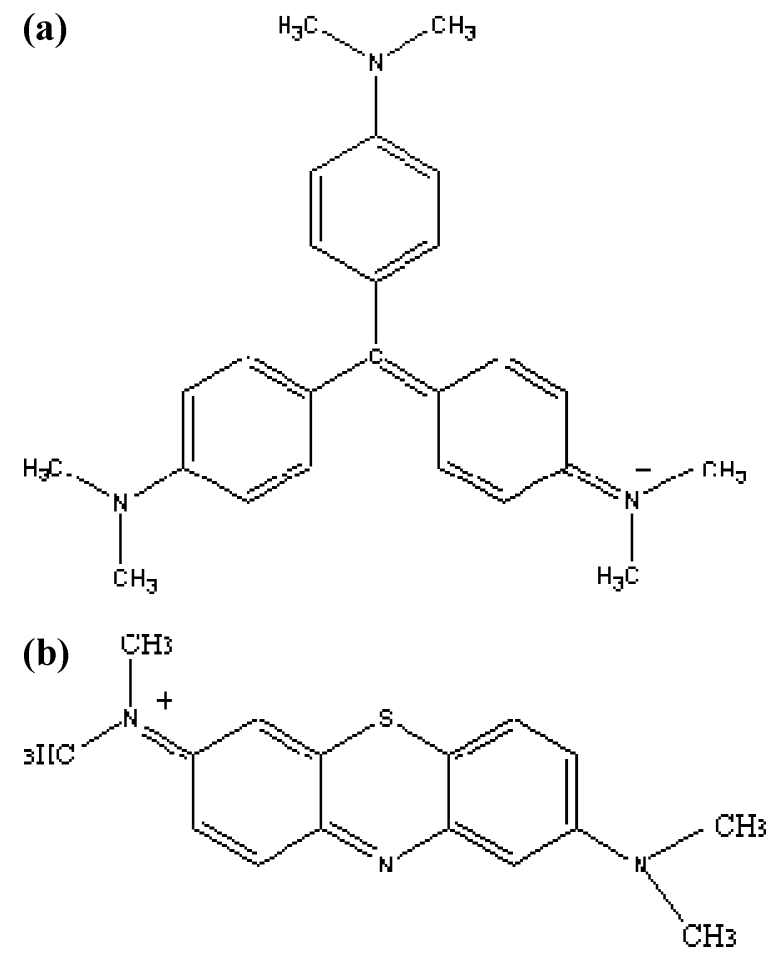

Figure 6. 6. Structures of (a) crystal violet and (b) methylene blue.

surface turns out to be isotropic. This fact suggests that $\mathrm{CV}^{+}$lying over the $\mathrm{SiO}^{-}$site may be aligned randomly; i.e., the individual tilt angle changes freely. It is reasonable while considering the resonance structure of $\mathrm{CV}^{+}$. As depicted in Figure 6 , the $\mathrm{CV}^{+}$ cation has a symmetric structure of $D_{3 h}$ with a size of $\sim 120 \AA^{2}$, different from a linear structure of methylene blue. As the positive charge on the $\mathrm{N}$ atom delocalizes around the whole planar structure through the $\pi$-electrons of phenylene ring, the structure becomes stabilized with a minimum energy calculated. ${ }^{44,47,48}$ Therefore, the $\mathrm{CV}^{+}$cation may approach toward the charged site with a binding angle varying in time and space. The average tilt angle then increases with increasing concentration and the contribution by the type II site interaction becomes significant (Figure 5), since the saturation surface density for the type II site is 4 times larger. The binding angles of $\mathrm{CV}^{+}$toward the type II sites are apparently restricted by repulsive forces exerted by both $\mathrm{CV}^{+}$cations and $\mathrm{SiOH}$ groups. The individual type II silanol sites are grouped closely together, and thus, the repulsion between $\mathrm{CV}^{+}$and the adjacent $\mathrm{CV}^{+}$cannot be ignored. With increased concentration, $\mathrm{CV}^{+}$binding on the type II surface tends to separate away from the neighboring adsorbates and align laterally to minimize the electrostatic repulsion. That may explain why the tilt angle increases with concentration. Figure 7 presents a pictorial description for the $\mathrm{CV}^{+}$adsorption on the type I and II sites.

Our results differ from those reported by Xu and co-workers, ${ }^{12}$ especially the argument for the $\mathrm{CV}^{+}$alignment on the type I surface. They obtained two different orientation factors for the type I and II species by using a different polarization combination of excitation and detection in the SHG technique. These factors are related to the orientation angles. Unfortunately, due to the lack of knowledge of the hyperpolarizability tensor matrix of $\mathrm{CV}^{+}$,

(47) Matsuoka, Y.; Yamaoka, K. Bull. Chem. Soc. Jpn. 1979, 52, 2244-2249. (48) Matsuoka, Y. J. Phys. Chem. 1980, 84, 1361-1366. 
(a)

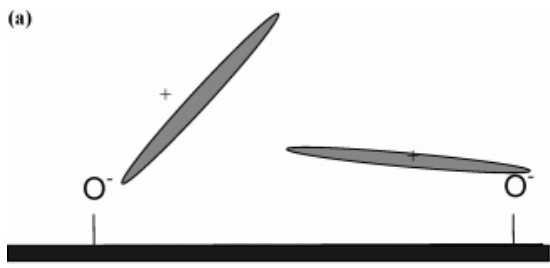

(b)

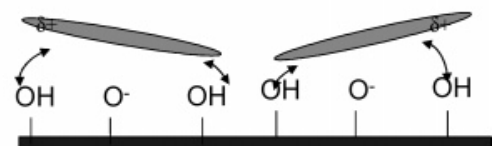

Figure 7. 7. Adsorption of $\mathrm{CV}^{+}$over the top of (a) the type $\mathrm{ISiO}^{-}$ sites and (b) the type $\mathrm{II} \mathrm{SiO}^{-}$sites.

they cannot determine the absolute orientation angle for $\mathrm{CV}^{+}$. Accordingly, they proposed that $\mathrm{CV}^{+}$with the positive charge localized at the central $\mathrm{C}$ atom of the planar structure can bind with the $\mathrm{SiO}^{-}$site firmly, lying flat on the top of the type I site. The silanol groups are separated far from each other $\left(>120 \AA^{2}\right)$ so that $\mathrm{a} \mathrm{CV}^{+}$cation can be placed flatly. In addition to the alignment difference, our work suggests that this class of silanol groups should be surrounded by an empty space even larger by a factor of 4 times. As for the $\mathrm{CV}^{+}$interaction with the type II sites, $\mathrm{Xu}$ and co-workers expected that $\mathrm{CV}^{+}$may be repelled from the $\mathrm{SiO}^{-}$site by the dipole field of the adjacent $\mathrm{SiOH}$ groups, thereby yielding weaker adsorption with a small angle tilted from the substrate surface. Our work further verifies that the tilt angle does not remain constant, but increases with the concentration. Because the positive charge on $\mathrm{CV}^{+}$is delocalized, we believe that more flexibility exists to pile up $\mathrm{CV}^{+}$on the top of the $\mathrm{SiO}^{-}$ site. The tilt angle is adjusted to minimize the repulsive hindrance from both the adjacent $\mathrm{SiOH}$ and $\mathrm{CV}^{+}$.

By probing a water monolayer on atomically smooth $a-\mathrm{SiO}_{2}$ with a similar EW-CRDS technique, Aarts el al. ${ }^{18}$ revealed three distinct structures of the silica surface. The first two are assigned to (100)-like faces of cristobalite, in which silanols are grouped closely to form the H-bonded networks. These two structures have been classified as vicinal silanol groups in the introduction. They are characterized by two orthogonally oriented species, having transition moments nearly aligned or nearly perpendicular to the surface. The third type with a much lower coverage is assigned to a (111)-like face, showing comparable s- and p-polarized absorption. We believe that this one should correspond to the isolated silanol group studied in this work. Different surface smoothness and probe methods may account for the different information obtained. Despite the lack of knowledge of the vicinal silanol groups, the $\mathrm{CV}^{+}$probe can thoroughly characterize the isolated silanol group.

\section{CONCLUSION}

We have used evanescent-wave cavity ring-down spectroscopy to characterize indirectly the silanol groups on a planar fusedsilica surface by investigating the response when the surface is exposed to and comes to equilibrium with different concentrations of crystal violet in acetonitrile. The polarization dependence of $\mathrm{CV}^{+}$adsorption at the $\mathrm{CH}_{3} \mathrm{CN}$ / silica interface is measured, from which we concluded that two types of isolated silanol groups exist on the surface, in agreement with previous work in the literature. The $\mathrm{CV}^{+}$cations may approach the first type of silanol sites with a binding angle aligned randomly, whereas $\mathrm{CV}^{+}$cations on the second type of silanol sites are repelled from the $\mathrm{SiO}^{-}$site, causing weaker adsorption and more restricted orientation. As the concentration increases, the $\mathrm{CV}^{+}$cations bound to the second type of silanol sites tend to lie with a larger tilt angle off the surface normal so that the repulsion from nearby $\mathrm{CV}^{+}$and $\mathrm{SiOH}$ may be reduced. The results obtained in this work agree qualitatively with those reported by $\mathrm{Xu}$ and co-workers using a SHG method but disagree quantitatively. We offer some explanations that might account for these discrepancies.

\section{ACKNOWLEDGMENT}

H.F.F. thanks the National Science Council for financial support to visit with R.N.Z.'s group. Part of this work is supported by National Science Council of Republic of China under Contract NSC 94-2113-M-002-014 (H.-F.F. and K.-C.L) and another part of this work is supported by the U.S. National Science Foundation.

Received for review December 17, 2006. Accepted March 12, 2007.

AC062386N 